\title{
Probing the diversity of healthy oral microbiome with bioinformatics approaches
}

\author{
Ji-Hoi Moon ${ }^{*} \mathcal{E}$ Jae-Hyung Lee \\ Department of Maxillofacial Biomedical Engineering, School of Dentistry, and Department of Life and Nanopharmaceutical Sciences, \\ Kyung Hee University, Seoul 02447, Korea
}

The human oral cavity contains a highly personalized microbiome essential to maintaining health, but capable of causing oral and systemic diseases. Thus, an in-depth definition of "healthy oral microbiome" is critical to understanding variations in disease states from preclinical conditions, and disease onset through progressive states of disease. With rapid advances in DNA sequencing and analytical technologies, population-based studies have documented the range and diversity of both taxonomic compositions and functional potentials observed in the oral microbiome in healthy individuals. Besides factors specific to the host, such as age and race/ethnicity, environmental factors also appear to contribute to the variability of the healthy oral microbiome. Here, we review bioinformatic techniques for metagenomic datasets, including their strengths and limitations. In addition, we summarize the interpersonal and intrapersonal diversity of the oral microbiome, taking into consideration the recent large-scale and longitudinal studies, including the Human Microbiome Project. [BMB Reports 2016; 49(12): 662-670]

\section{INTRODUCTION}

The human microbiota (the collection of microbes that live on and inside us) consists of a wide range of microorganisms whose aggregate membership exceeds the human somatic and germ cells by at least an order of magnitude $(1,2)$. The collection of genes in the microbiota is called the human microbiome (2); however, "microbiota" and "microbiome" are often used interchangeably (3). One of the most clinically relevant microbial habitats, the human oral cavity is colonized

*Corresponding authors. Jae-Hyung Lee, Tel: +82-2-961-9290; Fax: +82-2-962-0598; E-mail: jaehlee@khu.ac.kr, Ji-Hoi Moon, Tel: +82-2-961-0795; Fax: +82-2-962-0598; E-mail: prudence75@ khu.ac.kr

https://doi.org/10.5483/BMBRep.2016.49.12.164

Received 23 September 2016

Keywords: Bioinformatics, Diversity, Human Microbiome Project (HMP), Next-generation Sequencing (NGS), Oral microbiome by a personalized set of microorganisms, including bacteria, archaea, fungi, and viruses (4). Under healthy conditions, the oral microbiota lives in harmony with the host, similar to other body sites. The host provides an environment wherein the microbiome flourish, in turn keeping their host healthy (5). Conversely, the oral microbiome is also considered a key cause of oral diseases, including dental caries and periodontal diseases, as well as many systemic diseases such as diabetes and cardiovascular diseases $(5,6)$. Because of its crucial role in oral and systemic health, the oral microbiome has become an essential part of microbiomics.

An in-depth definition of a healthy microbiome is an indispensable step toward detecting significant variations in disease states and pre-clinical conditions, as well as understanding the disease onset and progression (7). The advent of next generation sequencing (NGS) or high-throughput sequencing has revolutionized the field of microbiome analysis, providing the tools necessary to address the issue (8). This prompted the launch of the NIH's Human Microbiome Project (HMP), constructed as a large, genome-scale community research project (NIH HMP Working Group, 2009). Over 200 healthy adults were enrolled, and samples were collected from 15 to 18 body sites, including oral, stool, skin, nasal, and vaginal areas, over a period of 1 to 3 visits (9). Besides two major scientific reports $(9,10)$, several companion papers analyzed the HMP oral datasets $(7,11-13)$, revealing great variability of the oral microbiome among and within healthy individuals. Furthermore, other recent large-scale and longitudinal studies have augmented our view of the oral microbiome, beyond that of the HMP.

In this paper, we review bioinformatic techniques for metagenomic datasets, including microbial community profiling, and highlight the strengths and weaknesses of the experimental approaches. We also summarize important findings that lead to the current understanding of the the range of healthy microbial diveristy. Although viruses, fungi, archaea and protozoa form a part of the normal microbiome (4), majority of the research is concentrated on the domain Bacteria. Therefore, we will focus exclusively on oral bacteria in this review. 


\section{BIOINFORMATIC ANALYSIS OF MICROBIOME SEQUENCE DATA}

Two distinct metagenomics approaches are commonly used: marker gene metagenomics and full shotgun metagenomics. Marker gene metagenomics is a fast and cost-effective way to obtain a taxonomic distribution profile. In this approach, specific regions of evolutionarily conserved marker genes are first amplified by PCR, and subsequently sequenced (14). In the case of bacterial (and/or archaeal) community analysis, the target region usually contains the $16 \mathrm{~S}$ ribosomal RNA (rRNA) gene (15). Hence, the approach is referred to as $16 \mathrm{~S}$ rRNA profiling. Full shotgun metagenomics, also referred as metagenomic whole genome sequencing (WGS), does not target a specific locus or marker gene, but instead breaks the isolated metagenomic DNA into smaller fragments, and subsequently sequences the individual pieces (14). The sequenced small fragments (i.e., sequencing raw reads) can be used for taxonomy profiling (who is there?) as well as for functional profiling (what are they doing?) (14). In this section, we briefly describe the scheme of the techniques involved and the bioinformatic pipelines, to analyze microbiome sequence data obtained from the above two methods.

\section{S rRNA profiling}

The 16S rRNA gene was introduced as a marker for bacterial phylogeny by Woese et al. (16). Ever since, it has been the gold standard for phylogenetic analysis of microbial communities and bacterial taxonomy (11). Bacterial 16S rRNA genes usually include nine hypervariable regions (V1-V9) that exhibit substantial sequence diversity among different bacterial species (17). Numerous studies have assessed the 16S rRNA gene and selected the most appropriate conserved regions to generate amplicons using universal primers, as well as identified the most effective hypervariable regions to be targeted (17-23). Unfortunately, no single hypervariable region is sufficiently different to define all bacterial species and a bias can be introduced by primer specificity as well as efficiency. Basically, the 16S rRNA profiling can be summarized into three steps: (1) preprocessing and denoising of raw reads, (2) taxonomic assignment, and (3) evaluation of microbial diversity.

(1) Preprocessing and denoising of raw sequencing reads:
Although there are standard operations and protocols to generate the NGS sequencing libraries, stochastic errors in the biological processes for the library creation, and/or incomplete chemical reactions in sequencing, could affect the overall quality of the sequencing library and sequencing datasets. Therefore, raw sequencing reads generated should be carefully checked for the successful downstream analysis in the preprocessing step. A number of computational tools have been used for the preprocessing: FastQC (http://www.bioinformatics. babraham.ac.uk/projects/fastqd) provides a quick quality check by running a modular set of analyses such as "per base sequence quality", "per sequence quality score", "sequence length distribution", "adapter content", etc.; FASTX- toolkit (http://hannonlab.cshl.edu/fastx toolkit/) allows detecting and trimming the low quality region of the individual read (especially 3 '-end of the reads); DUST is used to remove low-complexity regions in the sequencing read (24). Intrinsically, NGS techniques can harbor various errors in the sequencing reads, such as imprecise signals from longer homopolymer runs and chimera sequences. In the denoising step, these errors were identified and corrected for the accurate taxonomic assignments of the sequencing reads. Many popular software, such as QIIME (25) and mothur (26), have implemented the denoising algorithms. In particular, UCHIME is designed to detect chimeric sequences by comparing reference sequences to a database, or by performing de novo classification (clustering) (27). Preprocessed and denoised raw sequencing reads are subsequently subject to the process of taxonomic assignment.

(2) Taxonomic assignment: NGS allows investigators to detect and identify novel bacteria that have previously gone undetected. Subsequently, the assignment of $16 \mathrm{~S}$ rRNA read sequences originated from uncultured bacterial genome into a specific bacterial taxonomy is even more difficult. In two frequently used methods, the reads are assigned into bins, according to either homology between the reads and known reference sequences (i.e., phylotyping) or homology between the reads (i.e., operational taxonomic units [OTUs]) (28). The former method relies upon aligning reads with the reference 16S rRNA database using sequence alignment algorithms, such as BLAST (29). Besides NCBI Genbank, a number of rRNA databases have been constructed and used for the taxonomic assignment (Table 1). Each database has its own

Table 1. A list of $16 \mathrm{~S}$ ribosomal RNA database

\begin{tabular}{lll}
\hline \multicolumn{1}{c}{ Name } & 16S rRNA coverage & \multicolumn{1}{c}{ Database URL (reference) } \\
\hline CORE & Human Oral Bacteria & http://microbiome.osu.edu/ (32) \\
RDP & Archaea and Bacteria & https://rdp.cme.msu.edu/ (33) \\
Human Oral Microbiolome Database & Human Oral Bacteria & http://www.homd.org/index.php (65) \\
EzTaxon-e & Archaea and Bacteria & $\mathrm{http}: / / \mathrm{www}$.ezbiocloud.net/eztaxon (95) \\
SILVA & Archaea and Bacteria & $\mathrm{https:/www.arb-silva.de/} \mathrm{(96)}$ \\
Greengenes & Archaea and Bacteria & $\mathrm{http}: / /$ greengenes.secondgenome.com/ (97) \\
\hline
\end{tabular}


criteria for the curation of data from the original resources. For example, the Human Oral Microbiome Database (HOMD) (30) and CORE (31) have been constructed using 16S rRNA sequences exclusively from human oral bacteria. The second approach is to group $16 \mathrm{~S}$ rRNA sequencing reads into bins called OTUs with distance-based agglomerative clustering methods, such as CD-HIT (32) and UCLUST (33). Defining species by $97 \%$ identity in $16 \mathrm{~S}$ rRNA gene sequence is a commonly used criterion, but these distinctions are still controversial $(11,34)$.

NGS platforms generate massively greater number of reads compared to the classical Sanger sequencing, while the reads are relatively much shorter. Unfortunately, current databases and methods are not able to assign all species names or provide enough phylogenetic information for the billions of sequence reads (11). For example, the most commonly used tool for assigning taxonomy, the Ribosomal Database Project (RDP) Classifier (35), does not assign taxonomic names below the genus level $(11,36)$. Moreover, as revealed in our previous study, the RDP shows insufficient resolution for classifying the GN02 and Synergistetes (37). To supplement the limited phylogenetic information, 16S rRNA sequences are usually grouped using the OTU approach described above. Huse et al. (11) explored the HMP oral microbiota from over 200 individuals, and identified between 857 and 4,216 OTUs (Table 2). In terms of community membership, oral microbiota was especially diverse, showing the highest estimate of total richness after stool microbiota. Notably, richness as measured by the V1-V3 primers, was consistently higher than that measured by V3-V5 (11). In addition, some taxa (e.g. Lactobacilli OTUs) resolved better with V1-V3 while others (e.g. Bifidobacteriaceae OTUs) were better resolved with
V3-V5 (11). These differences may be due to a mismatch of the primers for amplification, or an inability to differentiate the taxon in that region of the rRNA gene (11). Therefore, as with all $16 \mathrm{~S}$ rRNA sequencing projects, diversity measurements should be compared with other results using the same $16 \mathrm{~S}$ rRNA gene region, and the presence of primer bias should not be ignored (11). Additionally, sequencing errors will also affect the taxonomic assignments of the sequence reads, possibly resulting in spurious OTUs and exaggerated diversity estimates, thus making direct comparisons between studies problematic (12).

(3) Evaluation of microbial diversity: To understand the structure and dynamics of microbial community, the measurement of diversity is essential. Two diversity measurements are frequently used to assess and compare microbial communities: alpha (or within-sample) diversity and beta (or betweensample) diversity. Alpha diversity is usually characterized using the total number of organisms within a sample (richness, which may be measured as the number of OTUs), the relative abundances of the organisms (evenness), or indices that combine these two dimensions. In contrast, beta diversity, is often characterized using the number of species (or OTUs) shared between two communities. In particular, UniFrac, a robust method for comparing the differences between microbial communities between samples, measures the proportion of shared branch lengths on a phylogenetic tree between samples $(3,38)$. Principal Coordinates Analysis (PCoA) can summarize and visualize the UniFrac distances between samples in a scatterplot where points (representing samples) that are more distant from one another are more dissimilar.

Table 2. Counts of patients included, OTUs and estimated richness (number of species) found for both the V1-V3 and the V3-V5 regions (11)

\begin{tabular}{|c|c|c|c|c|c|c|}
\hline \multirow{2}{*}{ Body Site } & \multicolumn{3}{|c|}{ V1-V3 } & \multicolumn{3}{|c|}{ V3-V5 } \\
\hline & Patients & OTUs & Estimated richness ${ }^{\mathrm{a}}$ & Patients & OTUs & Estimated richness $^{\mathrm{a}}$ \\
\hline Buccal mucosa & 114 & 2,025 & 6,635 & 198 & 898 & 4,650 \\
\hline Hard palate & 112 & 1,741 & 3,793 & 190 & 912 & 3,125 \\
\hline Keratinized gingiva & 117 & 1,545 & 4,387 & 206 & 857 & 3,352 \\
\hline Palatine Tonsils & 119 & 3,683 & 10,023 & 204 & 1,633 & 9,020 \\
\hline Saliva & 99 & 2,341 & 6,546 & 181 & 1,399 & 6,801 \\
\hline Subgingival plaque & 119 & 4,216 & 14,410 & 204 & 1,672 & 11,501 \\
\hline Supragingival plaque & 121 & 3,851 & 11,154 & 205 & 1,587 & 8,254 \\
\hline Throat & 110 & 2,343 & 5,601 & 192 & 1,136 & 4,154 \\
\hline Tongue dorsum & 119 & 3,651 & 7,910 & 205 & 1,503 & 7,947 \\
\hline Posterior fornix ${ }^{\mathrm{b}}$ & 59 & 428 & 1,151 & 95 & 400 & 1,466 \\
\hline Stool $^{b}$ & 118 & 6,050 & 23,665 & 209 & 5,391 & 33,627 \\
\hline
\end{tabular}

a Upper and lower confidence limits are not included in this table. ${ }^{b}$ Example of extraoral sites. The stool samples have the highest estimate of total richness, followed by the oral samples, particularly the plaque and tonsils. The skin sites, such as posterior fornix, have the lowest estimated richness. 


\section{Metagenomic WGS data analysis}

Although the $16 \mathrm{~S}$ rRNA profiling is a powerful, effective and straightforward technique to study microbial communities, it only provides the taxonomic composition. The metagenomic WGS data can provide not only taxonomy, but also the biological functional profiles for the microbial communities. The principles of taxonomy profiling processes that employ WGS data are similar to those described above. This section will therefore focus on the functional profiling of the microbial community. The analysis pipeline can be divided into four stages: (1) preprocessing, (2) reconstruction of raw sequencing reads (assembly), (3) gene prediction, and (4) functional annotations.

(1) Preprocessing: Preprocessing assesses the overall quality of WGS data, and most steps are similar to 16S rRNA profiling. Additionally, raw metagenomic NGS reads associated with a host (e.g. human) are checked for host DNA contamination, and the contaminated sequencing reads are removed. Fast short read mapping tools, such as BWA (39) and Bowtie 2 (40), are used to detect the contaminated sequencing reads by aligning raw sequencing reads against the host genome (e.g. human genome).

(2) Reconstruction of raw sequencing reads (assembly): The metagenomic WGS technique generates raw sequencing reads from the whole microbial genomes in the microbial community. Thus, to identify the specific genomes and/or complete protein coding genes in the genomes accurately, it is helpful to reconstruct the microbial genomes from raw sequencing reads. However, obtaining complete genomes has been challenging not only because of the highly repetitive DNA sequences abundant in a broad range of species (from bacteria to mammals), but also because of short reads and high data volumes produced by NGS technology. Therefore, an assembly of shorter reads into genomic contigs and their orientation into scaffolds is often performed. Most of the metagenomic WGS read assembly tools are designed and implemented based on the de Bruijn graph theory algorithm. Initially, all sequencing reads are fragmented into k-mers, following which they are used as the edges in the de Bruijn graph. The nodes of (k-1)-mer prefix and suffix are linked by the edges of k-mers for the graph. Finally, the assembler identifies the Eulerian paths that go across all edges just once in the graph (41). Velvet (42), ABySS (41) and SOAPdenovo (44) use the de Bruijn graph to assemble whole metagenomes from raw sequencing reads. In the HMP, the raw sequencing reads from 749 metagenomic samples were successfully used to assemble contigs using an optimized SOAPdenovo protocol (8). Recently, more sophisticated algorithms have been developed and applied to the next-generation assemblers, such as Meta-IDBA (45), MetaVelvet-SL (46) and IDBA-UD (47).

(3) Gene prediction: Following reconstruction, next stage is to identify genes in the reads or assembled contigs and/or scaffolds. The prediction of genes in metagenomic contents is still a fairly difficult problem, although several gene prediction algorithms have been successfully employed for prokaryotic genomes. To predict genes in metagenomic studies, especially for de novo genes, several computational methods have been developed, including MetaGeneMark (48), MetaProdigal (49), Glimmer-MG (50), and FragGeneScan (51). Notably, the performance of gene-predicting tools varies considerably: in a comparison of five widely used ab initio gene-calling algorithms including FragGeneScan and MetaGeneMark, FragGeneScan is rather accurate for predicting reading frames on short raw reads (75-1,000 bp) while other tools, such as MetaGeneMark, are better suited for higher-quality sequences such as assembled contigs (52). Moreover, it has been reported that combining predictions from various programs can improve the accuracy of prediction and annotation of metagenomic reads (53). Accordingly, researchers should carefully decide what tools to use in their metagenomic study, which potentially impacts the results and conclusion.

(4) Functional annotations: After gene prediction, the identified genes are functionally annotated by comparing the known genes in the functional annotation databases such as PFAM (54), IMG/M (55), COG (56) and MetaRef (57). Further analysis of the relationship between the microbiome and the host phenotype is performed using metabolic pathway information database, i.e. KEGG (58), eggNOG (59) and MinPath (60). In the part of the HMP, Abubucker et al. devised HMP Unified Metabolic Analysis Network (HUMAnN) to construct metabolic networks of the microbial communities (61). In this study, raw sequencing reads were searched against protein sequence databases, and HUMAnN recovers the abundances of individual orthologues gene families and pathway. More specifically, MBLASTX, KEGG orthology and MinPath have been used to assign genes and available pathways. Recently, several metagenomic analysis pipeline software have been developed, such as MG-RAST (62) and IMG/MER (https://img.jgi.doe.gov/cgi-bin/mer/main.cgi). The pipelines provide the functional annotation modules in their fully automated pipeline web-server and thus, researchers can easily perform functional annotation tasks using their own data in the web (15).

\section{COMPOSITION AND DIVERSITY OF ORAL MICROBIOME}

The HMP assessed oral microbiome composition of seven intra oral sites (buccal mucosa, hard palate, keratinized gingiva, saliva, sub- and supra gingival plaque, and tongue dorsum) and two oropharyngeal sites (throat and palatine tonsils) from 182-206 healthy subjects (18 to 40 years old). A total of 185-322 genera belonging to 13-19 bacterial phyla were discovered (13). The dominant phyla were Firmicutes, Bacteroidetes, Proteobacteria, Fusobacteria and Actinobacteria, accounting for over $95 \%$ of the entire oral microbiome. An individual sample from a single site of a single subject contained 23-50 genera from 6-9 phyla (13). Among all body 
habitats, the oral habitats have the highest alpha diversity showing the highest OTU level richness, after the stool samples (Table 2), while the skin and vaginal microbiota show lower alpha diversity $(11,13)$. Comparing samples from the same sites among subjects (beta diversity), the oral sites have the lowest beta diversities, signifying that members of the population shared relatively similar organisms in oral sites than in other body sites (10). However, the HMP oral datasets also emphasize the high variance across individuals, especially at the sub-genus level: even OTUs existing in almost every subject, or dominating in some samples, showed variation in relative abundance (11). In the following sections, we discuss in detail about the specific factors that contribute to the variability of the healthy oral microbiome.

\section{Different oral biogeographic niches}

The oral cavity is a humid environment which is kept at a fairly consistent temperature $\left(34^{\circ} \mathrm{C}\right.$ to $\left.36^{\circ} \mathrm{C}\right)$ and a relatively neutral $\mathrm{pH}$ in most areas, and thus provide great condition for the growth of various microorganisms (63). The oral cavity is composed of diverse habitats with different anatomical structures and physicochemical factors. The oral mucosa covers the cheek, tongue, gingiva, palate, and floor of the mouth and allows rapid elimination of adhering bacteria due to a continuous shedding of its surface epithelial cells (63). On the other hand, papillary surface of the tongue provides shelter for adhering bacteria and protects these bacteria from mechanical cleaning. The hard surface of teeth offers many sites for bacterial colonization, in both supra- and subgingival areas. The gingival crevice (area between the junctional epithelium of the gingiva and teeth), provides a distinctive microbial colonization site, consisting of both hard and soft tissues (63). The epithelium may be keratinized (palate) or nonkeratinized (gingival crevice). Hence, the oral cavity is not considered a uniform environment.

The HMP revealed a substantial divergence in the species richness and evenness among different oral habitats, and also identified microorganisms with specific niche preferences. Hard palate showed the lowest total richness, however the gingival plaque showed the highest total richness (11) (Table 2). Oral sites, especially saliva, have the highest evenness, while buccal mucosa and keratinized gingiva have lower alpha diversity than the other oral sites $(10,13)$. Each oral habitat in almost every subject was characterized by one or a few signature taxa making up the plurality of the community with highly variable relative abundance among both the individuals and the oral habitats. Most oral habitats are dominated by Streptococcus, followed in abundance by Haemophilus in the buccal mucosa, Actinomyces in the supragingival plaque, and Prevotella in the subgingival plaque $(10,13)$. There is an overlap of species detected in almost all oral sites, such as certain species of Streptococcus (OTUs \#2, 5 and 6), Gemella (OTUs \#7 and 8), Granulicatella (OTU \#13), Fusobacterium (OTUs \#9 and 27), and Veillonella
(OTUs \#4 and 7) (11). However, several abundant genera had multiple OTUs with distinct preferences for often only one or two oral sites, such as Bacteroides, Prevotella, Corynebacterium, Fusobacterium, Pasteurella, and Neisseria (11). For example, Corynebacterium matruchotii (OTU \#15) existed almost only in the supragingival plaque, while Corynebacterium argentoratense (OTU \#188) was mostly found in saliva and to a lesser extent on the hard palate (11). This may be due to the desquamation of the epithelial cells and the shear forces that are generated during chewing in the buccal fold and the hard palate (64). Analysis of oral samples collected from the elderly (range 73-93), Lautropia mirabilis was significantly associated with the supragingival plaque, while Treponema socranskii was found only in the subgingival plaque (65). This may be explained by the low oxidation-reduction potential of the subgingival plaque. In the oropharynx, the distribution of Firmicutes, Proteobacteria, and Bacteroidetes was similar to that in saliva, but had more Proteobacteria than found in the mouth (66).

\section{Influence of geography, climate and ethnicity}

Although the HMP produced a huge volume of data, the resulting 16S rRNA datasets are composed of samples from medical students in the USA, and host information is nearly prohibitive to access, which lead to removal of the potential to observe any systematic patterns and regional or ethnic differences (67). A population-scale study of 120 healthy individuals from 12 worldwide locations showed a significant variation in the saliva microbiome according to the locations (68). Notably, the saliva microbiome of Batwa Pygmies, a former hunter-gatherer group from Africa, was much more diverse than the saliva microbiome of two agricultural African groups, probably owing to their different lifestyle and diet (69). In another study of 3 human groups from different geographic and climatic areas (76 native Alaskans, 10 Germans and 66 Africans) the distinctiveness of the saliva microbiome was seen, the reasons of which (e.g. different lifestyles and/or host genetics and physiology) remain to be clarified (70). In the study, alpha diversity was highest for the German group and lowest for the African group, while the opposite was true for beta diversity. It is intriguing to speculate that the higher population density of Germany may provide more opportunities for bacteria to be spread among individuals (70).

Ethnicity is likely to exert a selection pressure on the oral microbiome. Mason et al. (71) analyzed dental plaque and saliva samples collected from 192 subjects belonging to four ethnic affiliations (non-Hispanic blacks, non-Hispanic whites, Chinese, and Latinos) and found obvious ethnicity-specific clustering of microbial communities, thus reinforcing prior observations (72-74). It seems that this selection pressure is most likely genetic rather than environmental, because the two ethnicities that shared a common food, nutritional and lifestyle heritage (Caucasians and African Americans) showed substantial microbial diversity (71). It is known that not only 
innate immune responses to infectious agents, but also tooth morphologies, vary according to ethnic affiliation (75-78). Therefore, ethnicity possibly contribute to the bacterial selection by affecting the environment for bacterial colonization (71).

\section{Vertical and horizontal transmission}

Vertical transmission from mother to child starts at birth (79). Depending on the delivery mode (vaginal or Caesarian), infants acquire bacterial communities similar to their mother's vaginal microbiota or skin microbiota (80). A study of healthy three-month-old infants delivered vaginally (25 infants) and born by C-section (38 infants) found differences in the infant's oral microbiota owing to the mode of delivery, with vaginally delivered infants having a higher taxonomic diversity (81). The method of feeding also affects the infant's microbiome: oral lactobacilli with antimicrobial properties were found in breast-fed infants but not found in formula-fed infants $(82,83)$. Horizontal transmission of oral microbiota among siblings and other individuals sharing the same environment also contributes to oral microbiome diversity. In a study, 264 saliva samples were collected from 107 individuals (including 45 twin pairs), at up to three time-points during a 10-year period, spanning adolescence. The twins resembled each other more closely than the whole population at all time-points, but became less similar to each other when they aged and no longer cohabited (84).

\section{Temporal variation}

Studies looking at the temporal variation of the oral microbiome have revealed conflicting results: in a longitudinal study of 5 adults at three time-points (from 5 to 29 days), the salivary microbial community appeared to be stable at different time points (85). The HMP consortium (10) and Zhou et al. (13) reported that, among $22 \mathrm{HMP}$ habitats of the human body, the oral habitat has the most stable microbiota, showing the highest community similarity between two visits (mean time interval between visits is 212 days), while the skin and vaginal microbiota are less stable. In contrast, a reanalysis of the HMP datasets by a method for quantifying the difference between two cohorts revealed that the relative abundances of core OTUs in an individual sample showed significantly greater changes from $1^{\text {st }}$ to $2^{\text {nd }}$ visit at oral and stool body regions, as compared with the vaginal region (12). More recently, a weekly longitudinal study of 85 adults over 3 months, showed high levels of temporal variability in both diversity and community structure in tongue microbiome, as in other body habitats studied (86). Furthermore, this study found that the composition of an individual's microbiome as well as their degree of temporal variability, are personalized features. Collectively, although intrapersonal variation over time is lower than interpersonal variation, intrapersonal temporal dynamics need to be considered when attempting to link changes in microbiome structure to changes in health status (86).

\section{Age-related changes}

Along with a variety of physiological changes which accompanies aging, microbial habitats also greatly change in the oral cavity. The eruption of primary teeth and replacement of the primary dentition with permanent dentition may lead to shifts in the microbial community composition at different phases of life (87). Edentulous infants have been found to have lower diversity than their mothers or primary care givers in the oral microbial composition (88). In deciduous dentition, a higher proportion of Proteobacteria (Gammaproteobacteria, Moraxellaceae) was found than Bacteroidetes. With increasing age, Bacteroidetes (mainly genus Prevotella), Veillonellaceae family, Spirochaetes, and candidate division TM7 increased (89). Several organisms, including members of the genera Veillonella, Actinomyces and Streptococcus, were reported to have age-specific abundance profiles during adolescence (84). Xu et al. (87) analyzed the oral microbiome (saliva, supragingiva and mucosa) across a wide age range (3 days-76 years), in which only a very small overlap of shared OTU was observed. In this study, a distinct temporal shift was observed in the relative abundance of most genera. The average relative abundance of the dominant bacterial phyla, Actinobacteria, Bacteroides, Firmicutes, Fusobacteria, Proteobacteria, Spirochetes and candidate division TM7 varied by age/dentition stage (87).

\section{CONCLUSIONS}

We have only begun to understand the tremendous diversity of the oral microbiome and a number of challenges remain, such as the vast uncultivated species and the lack of reference genomes (90). Until recently, about half of all known bacterial phyla were identified only from their 16S rRNA gene sequences (91). In fact, the bacteria that can be grown in the laboratory are only a portion of the total diversity that exists in the oral cavity (92). One method to address this challenge is single-cell genomics, which is a powerful tool for accessing genetic information from uncultivated microorganisms (93). Future work combining metagenomics and single cell genomics, as well as advances in each separate method, should help to overcome these issues, providing new insights into the uncultivated lineages (94).

Rapidly developing sequencing methods and analytical techniques are enhancing our ability to understand the human microbiome, leading to the concept of a 'personal microbiome'. The focus now shifts from characterizing oral microbiota to functional studies encompassing genomics, transcriptomics, and metabolomics of both host and microbes. Future investigations will inevitably be personal omics profiling in order to probe the temporal patterns associated with both molecular changes and related physiological health and disease. This knowledge is vital for the development of efficacious prevention and treatment protocols for oral diseases and, ultimately, contribute to the development of personalized medicine and personalized dental medicine. 


\section{ACKNOWLEDGEMENTS}

This work was supported by the Ministry of Science, ICT \& Future Planning (NRF-2015R1C1A2A01054588) and the National Honor Scientist Program (NRF-2012R1A3A1050385). This work was also supported in part by 2016 Sabbatical Research Program of Kyung Hee University.

\section{REFERENCES}

1. Hamady M and Knight R (2009) Microbial community profiling for human microbiome projects: tools, techniques, and challenges. Genome Res 19, 1141-1152

2. Turnbaugh PJ, Ley RE, Hamady M, Fraser-Liggett CM, Knight R and Gordon JI (2007) The human microbiome project. Nature 449, 804-810

3. Ursell LK, Metcalf JL, Parfrey LW and Knight R (2012) Defining the human microbiome. Nutr Rev Suppl 1, S38-44

4. Wade WG (2013) The oral microbiome in health and disease. Pharmacol Res 69, 137-143

5. Zarco MF, Vess TJ and Ginsburg GS (2012) The oral microbiome in health and disease and the potential impact on personalized dental medicine. Oral Dis 18, 109-120

6. He J, Li Y, Cao Y, Xue J and Zhou X (2015) The oral microbiome diversity and its relation to human diseases. Folia Microbiol (Praha) 60, 69-80

7. Segata N, Haake SK, Mannon P et al (2012) Composition of the adult digestive tract bacterial microbiome based on seven mouth surfaces, tonsils, throat and stool samples. Genome Biol 13, R42

8. Gevers D, Pop M, Schloss PD and Huttenhower C (2012) Bioinformatics for the Human Microbiome Project. PLoS Comput Biol 8, e1002779

9. Human Microbiome Project Consortium (2012b) A framework for human microbiome research. Nature 486, 215-221

10. Human Microbiome Project Consortium (2012a) Structure, function and diversity of the healthy human microbiome. Nature 486, 207-214

11. Huse SM, Ye Y, Zhou $Y$ and Fodor AA (2012) A core human microbiome as viewed through 16S rRNA sequences clusters. PLoS One 7, e34242

12. Li K, Bihan M and Methé BA (2013) Analyses of the stability and core taxonomic memberships of the human microbiome. PLoS One 8, e63139

13. Zhou Y, Gao H, Mihindukulasuriya KA et al (2013) Biogeography of the ecosystems of the healthy human body. Genome Biol 14, R1

14. Sharpton TJ (2014) An introduction to the analysis of shotgun metagenomic data. Front Plant Sci 5, 209

15. Oulas A, Pavloudi C, Polymenakou P et al (2015) Metagenomics: tools and insights for analyzing next-generation sequencing data derived from biodiversity studies. Bioinform Biol Insights 9, 75-88

16. Woese CR and Fox GE (1977) Phylogenetic structure of the prokaryotic domain: the primary kingdoms. Proc Natl Acad Sci U S A 74, 5088-5090
17. Mizrahi-Man O, Davenport ER and Gilad Y (2013) Taxonomic classification of bacterial 16S rRNA genes using short sequencing reads: evaluation of effective study designs. PLoS One 8, e53608

18. Wang Q, Garrity GM, Tiedje JM and Cole JR (2007) Naive Bayesian classifier for rapid assignment of rRNA sequences into the new bacterial taxonomy. Appl Environ Microbiol 73, 5261-5267

19. Huse SM, Dethlefsen L, Huber JA, Mark Welch D, Relman DA and Sogin ML (2008) Exploring microbial diversity and taxonomy using SSU rRNA hypervariable tag sequencing. PLoS Genet 4, e1000255

20. Liu Z, DeSantis TZ, Andersen GL and Knight R (2008) Accurate taxonomy assignments from $16 \mathrm{~S}$ rRNA sequences produced by highly parallel pyrosequencers. Nucleic Acids Res 36, e120

21. Nossa CW, Oberdorf WE, Yang L et al (2010) Design of 16S rRNA gene primers for 454 pyrosequencing of the human foregut microbiome. World J Gastroenterol 16, 4135-4144

22. Claesson MJ, Wang Q, O'Sullivan O et al (2010) Comparison of two next-generation sequencing technologies for resolving highly complex microbiota composition using tandem variable $16 \mathrm{~S}$ rRNA gene regions. Nucleic Acids Res 38, e200

23. Tremblay J, Singh K, Fern A et al (2015) Primer and platform effects on $16 \mathrm{~S}$ rRNA tag sequencing. Front Microbiol 6, 771

24. Morgulis A, Gertz EM, Schäffer AA and Agarwala R (2006) A fast and symmetric DUST implementation to mask low-complexity DNA sequences. J Comput Biol 13, 1028-1040

25. Sevinsky JR, Turnbaugh PJ, Walters WA et al (2010) QIIME allows analysis of high-throughput community sequencing data. Nat Methods 7, 335-336

26. Schloss PD, Westcott SL, Ryabin T et al (2009) Introducing mothur: open-source, platform-independent, communitysupported software for describing and comparing microbial communities. Appl Environ Microbiol 75, 7537-7541

27. Edgar RC, Haas BJ, Clemente JC, Quince $C$ and Knight $R$ (2011) UCHIME improves sensitivity and speed of chimera detection. Bioinformatics 27, 2194-2200

28. Schloss PD and Westcott SL (2011) Assessing and improving methods used in operational taxonomic unit-based approaches for 16S rRNA gene sequence analysis. Appl Environ Microbiol 77, 3219-3226

29. Altschul SF, Madden TL, Schäffer AA et al (1997) Gapped BLAST and PSI-BLAST: a new generation of protein database search programs. Nucleic Acids Res 25, 3389-3402

30. Chen T, Yu WH, Izard J, Baranova OV, Lakshmanan A and Dewhirst FE (2010) The Human Oral Microbiome Database: a web accessible resource for investigating oral microbe taxonomic and genomic information. Database (Oxford) 2010:baq013

31. Griffen AL, Beall CJ, Firestone ND et al (2011) CORE: a phylogenetically-curated 16S rDNA database of the core oral microbiome. PLoS One 6, e19051

32. Fu L, Niu B, Zhu Z, Wu S and Li W (2012) CD-HIT: accelerated for clustering the next-generation sequencing 
data. Bioinformatics 28, 3150-3152

33. Edgar RC (2010) Search and clustering orders of magnitude faster than BLAST. Bioinformatics 26, 2460-2461

34. Schloss PD and Handelsman J (2005) Introducing DOTUR, a Computer Program for Defining Operational Taxonomic Units and Estimating Species Richness. Appl Environ Microbiol 71,1501-1506

35. Cole JR, Wang Q, Fish JA et al (2013) Ribosomal Database Project: data and tools for high throughput rRNA analysis. Nucleic Acids Res 42, D633-642

36. Kotamarti RM, Hahsler $M$, Raiford D, McGee $M$ and Dunham MH (2010) Analyzing taxonomic classification using extensible Markov models. Bioinformatics 26, 2235-2241

37. Moon JH, Lee JH and Lee JY (2015) Subgingival microbiome in smokers and non-smokers in Korean chronic periodontitis patients. Mol Oral Microbiol 30, 227-241

38. Lozupone C and Knight R (2005) UniFrac: a new phylogenetic method for comparing microbial communities. Appl Environ Microbiol 71, 8228-8235

39. Li H and Durbin R (2009) Fast and accurate short read alignment with Burrows-Wheeler transform. Bioinformatics 25, 1754-1760

40. Langmead B and Salzberg SL (2012) Fast gapped-read alignment with Bowtie 2. Nat Methods 9, 357-359

41. Kim Y, Koh I and Rho M (2014) Deciphering the human microbiome using next-generation sequencing data and bioinformatics approaches. Methods 79-80, 52-59

42. Zerbino DR and Birney E (2008) Velvet: algorithms for de novo short read assembly using de Bruijn graphs. Genome Res 18, 821-829

43. Simpson JT, Wong K, Jackman SD, Schein JE, Jones SJ and Birol I (2009) ABySS: a parallel assembler for short read sequence data. Genome Res 19, 1117-1123

44. Luo R, Liu B, Xie $Y$ et al (2012) SOAPdenovo2: an empirically improved memory-efficient short-read de novo assembler. Gigascience 1, 18

45. Peng Y, Leung HC, Yiu SM and Chin FY (2011) MetaIDBA: a de Novo assembler for metagenomic data. Bioinformatics 27, i94-101

46. Afiahayati, Sato K and Sakakibara Y (2015) MetaVelvet-SL: an extension of the Velvet assembler to a de novo metagenomic assembler utilizing supervised learning. DNA Res 22, 69-77

47. Peng Y, Leung HC, Yiu SM and Chin FY (2012) IDBA-UD: a de novo assembler for single-cell and metagenomic sequencing data with highly uneven depth. Bioinformatics $28,1420-1428$

48. Zhu W, Lomsadze A and Borodovsky M (2010) Ab initio gene identification in metagenomic sequences. Nucleic Acids Res 38, e132

49. Hyatt D, LoCascio PF, Hauser LJ and Uberbacher EC (2012) Gene and translation initiation site prediction in metagenomic sequences. Bioinformatics 28, 2223-2230

50. Kelley DR, Liu B, Delcher AL, Pop M and Salzberg SL (2012) Gene prediction with Glimmer for metagenomic sequences augmented by classification and clustering. Nucleic Acids Res 40, e9

51. Rho M, Tang $\mathrm{H}$ and $\mathrm{Ye} \mathrm{Y}$ (2010) FragGeneScan: predicting genes in short and error-prone reads. Nucleic Acids Res 38, e191

52. Trimble WL, Keegan KP, D'Souza M et al (2012) Short-read reading-frame predictors are not created equal: sequence error causes loss of signal. BMC Bioinformatics 13,183

53. Yok NG and Rosen GL (2011) Combining gene prediction methods to improve metagenomic gene annotation. BMC Bioinformatics 12, 20

54. Finn RD, Coggill P, Eberhardt RY et al (2015) The Pfam protein families database: towards a more sustainable future. Nucleic Acids Res 44, D279-285

55. Markowitz VM, Chen IM, Chu K et al (2014) IMG/M 4 version of the integrated metagenome comparative analysis system. Nucleic Acids Res 42, D568-573

56. Tatusov RL, Galperin MY, Natale DA and Koonin EV (2000) The COG database: a tool for genome-scale analysis of protein functions and evolution. Nucleic Acids Res 28, 33-36.

57. Huang K, Brady A, Mahurkar A et al (2014) MetaRef: a pan-genomic database for comparative and community microbial genomics. Nucleic Acids Res 42, D617-624.

58. Kanehisa M and Goto S (2000) KEGG: kyoto encyclopedia of genes and genomes. Nucleic Acids Res 28, 27-30

59. Huerta-Cepas J, Szklarczyk D and Forslund K (2016) eggNOG 4.5: a hierarchical orthology framework with improved functional annotations for eukaryotic, prokaryotic and viral sequences. Nucleic Acids Res 44, D286-293

60. Ye Y and Doak TG (2009) A parsimony approach to biological pathway reconstruction/inference for genomes and metagenomes. PLoS Comput Biol 5, e1000465

61. Abubucker S, Segata N, Goll J et al (2012) Metabolic reconstruction for metagenomic data and its application to the human microbiome. PLoS Comput Biol 8, e1002358

62. Keegan KP, Glass EM and Meyer F (2016) MG-RAST, a Metagenomics Service for Analysis of Microbial Community Structure and Function. Methods Mol Biol 1399, 207-233

63. Marcotte $\mathrm{H}$ and Lavoie MC (1998) Oral Microbial Ecology and the Role of Salivary Immunoglobulin A. Microbiol Mol Biol Rev 62, 71-109

64. Chen H and Jiang W (2014) Application of high-throughput sequencing in understanding human oral microbiome related with health and disease. Front Microbiol 5, 508

65. Preza D, Olsen I, Willumsen T, Grinde B and Paster B (2009) Diversity and site-specificity of the oral microflora in the elderly. Eur J Clin Microbiol Infect Dis 28, 1033-1040

66. Lemon KP, Klepac-Ceraj V, Schiffer HK, Brodie EL, Lynch SV and Kolter R (2014) Comparative analyses of the bacterial microbiota of the human nostril and oropharynx. MBio 1, e00129-10

67. McDonald D, Birmingham A and Knight R (2015) Context and the human microbiome. Microbiome 3,52

68. Nasidze I, Li J, Quinque D, Tang K and Stoneking M (2009) Global diversity in the human salivary microbiome. Genome Res 19, 636-643

69. Nasidze I, Li J, Schroeder R, Creasey JL, Li M and Stoneking M (2011) High Diversity of the Saliva Microbiome in Batwa Pygmies. PLoS One 6, e23352

70. Li J, Quinque D, Horz HP et al (2014) Comparative 
analysis of the human saliva microbiome from different climate zones: Alaska, Germany, and Africa. BMC Microbiol 14, 316

71. Mason MR, Nagaraja HN, Camerlengo $T$, Joshi $\mathrm{V}$ and Kumar PS (2013) Deep sequencing identifies ethnicityspecific bacterial signatures in the oral microbiome. PLoS One 8, e77287

72. Rylev M and Kilian M (2008) Prevalence and distribution of principal periodontal pathogens worldwide. J Clin Periodontol 35(8 Suppl), 346-361

73. Haffajee AD, Bogren A, Hasturk $H$, Feres $M$, Lopez NJ and Socransky SS (2004) Subgingival microbiota of chronic periodontitis subjects from different geographic locations. J Clin Periodontol 31, 996-1002

74. Kim TS, Kang NW, Lee SB, Eickholz P, Pretzl B and Kim CK (2009) Differences in subgingival microflora of Korean and German periodontal patients. Arch Oral Biol 54, 223-229

75. Miller MA and Cappuccio FP (2007) Ethnicity and inflammatory pathways-implications for vascular disease, vascular risk and therapeutic intervention. Curr Med Chem 14, 1409-1425

76. Nguyen DP, Genc M, Vardhana S, Babula O, Onderdonk A and Witkin SS (2004) Ethnic differences of polymorphisms in cytokine and innate immune system genes in pregnant women. Obstetrics and Gynecology 104, 293-300

77. Lavelle CL (1970) Crowding and spacing within the human dental arch of different racial groups. Arch Oral Biol 15, 1101-1103

78. Lavelle CL (1971) Mandibular molar tooth configurations in different racial groups. J Dent Res 50, 1353

79. Zaura E, Nicu EA, Krom BP and Keijser BJ (2014) Acquiring and maintaining a normal oral microbiome: current perspective. Front Cell Infect Microbiol 4, 85

80. Dominguez-Bello MG, Costello EK, Contreras $M$ et al (2010) Delivery mode shapes the acquisition and structure of the initial microbiota across multiple body habitats in newborns. Proc Natl Acad Sci U S A 107, 11971-11975

81. Lif Holgerson P, Harnevik L, Hernell O, Tanner AC and Johansson I (2011) Mode of birth delivery affects oral microbiota in infants. J Dent Res 90, 1183-1188

82. Holgerson PL, Vestman NR, Claesson R et al (2013) Oral microbial profile discriminates breast-fed from formula-fed infants. J Pediatr Gastroenterol Nutr 56, 127-136

83. Vestman NR, Timby N, Holgerson PL et al (2013) Characterization and in vitro properties of oral lactobacilli in breastfed infants. BMC Microbiol 13, 193

84. Stahringer SS, Clemente JC, Corley RP et al (2012)
Nurture trumps nature in a longitudinal survey of salivary bacterial communities in twins from early adolescence to early adulthood. Genome Res 22, 2146-2152

85. Lazarevic V, Whiteson $K$, Hernandez D, François $P$ and Schrenzel J (2010) Study of inter-and intra-individual variations in the salivary microbiota. BMC Genomics 11, 523

86. Flores GE, Caporaso JG, Henley JB et al (2014) Temporal variability is a personalized feature of the human microbiome. Genome Biol 15, 531

87. Xu X, He J, Xue J et al (2015) Oral cavity contains distinct niches with dynamic microbial communities. Environ Microbiol 17, 699-710

88. Cephas KD, Kim J, Mathai RA et al (2011) Comparative analysis of salivary bacterial microbiome diversity in edentulous infants and their mothers or primary care givers using pyrosequencing. PLoS One 6, e23503

89. Crielaard W, Zaura E, Schuller AA, Huse SM, Montijn RC and Keijser BJ (2011) Exploring the oral microbiota of children at various developmental stages of their dentition in the relation to their oral health. BMC Med Genomics 4, 22

90. McLean JS (2014) Advancements toward a systems level understanding of the human oral microbiome. Front Cell Infect Microbiol 4, 98

91. Lasken RS and McLean JS (2014) Recent advances in genomic DNA sequencing of microbial species from single cells. Nat Rev Genet 15, 577-584

92. Dewhirst FE, ChenT, Izard J et al (2010) The human oral microbiome. J Bacteriol 192, 5002-5017

93. Clingenpeel $\mathrm{S}$, Schwientek $\mathrm{P}$, Hugenholtz $\mathrm{P}$ and Woyke $\mathrm{T}$ (2014) Effects of sample treatments on genome recovery via single-cell genomics. ISME J 8, 2546-9

94. Solden L, Lloyd K and Wrighton K (2016) The bright side of microbial dark matter: lessons learned from the uncultivated majority. Curr Opin Microbiol 31, 217-226

95. Kim OS, Cho YJ, Lee K et al (2012) Introducing EzTaxon-e: a prokaryotic 16S rRNA gene sequence database with phylotypes that represent uncultured species. Int I Syst Evol Microbiol 62, 716-721

96. Quast C, Pruesse E, Yilmaz P et al (2013) The SILVA ribosomal RNA gene database project: improved data processing and web-based tools. Nucleic Acids Res 41, D590-596

97. DeSantis TZ, Hugenholtz P, Larsen N et al (2006) Greengenes, a chimera-checked 16S rRNA gene database and workbench compatible with ARB. Appl Environ Microbiol 72, 5069-5072 